\title{
A useful empirical Bayesian method to analyse industrial data from saturated factorial designs
}

\author{
Marta Yukie Baba ${ }^{a}$, Jorge Alberto Achcar ${ }^{\text {a,b }}$, Fernando Antonio Moala ${ }^{a}$, Sergio Minoru Oikawa \\ and Claudio Luis Piratelli ${ }^{\mathbf{b}^{*}}$
}

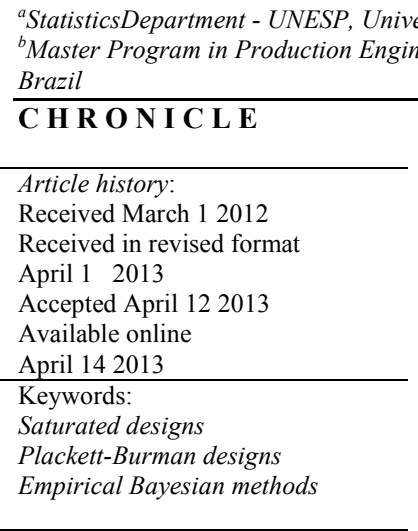

\section{Introduction}

Factorial designs have been extensively used by experimental researchers in many areas of interest such as agriculture, engineering, medical research, industrial research (Fisher, 1926,1935; Yates,1935,1937). Box et al. (1978) described the systematic exploration of factorial designs in industrial applications. Some important applications of factorial or fractional factorial experimental design techniques in manufacturing industries are presented in the literature. Philpott et al. (1996) employed a factorial design approach to identify key cost drivers of a process and to develop practical cost models from contract quotes. Kleijnen e Standridge (1988) used factorial designs to simulate a Flexible Manufacturing System. Feng et al. (2003) used a factorial design to illustrate the goodness of Neural Networks Modeling of Honing Surface Roughness Parameters defined by IS0 13565.

\footnotetext{
* Corresponding author. Tel.: +55 1633017309

E-mail: clpiratelli@uniara.com.br (C. L. Piratelli)

(C) 2013 Growing Science Ltd. All rights reserved. doi: $10.5267 /$ j.ijiec.2013.04.001
} 
Pei et al. (2003) employed a factorial design to reveal the main effects and the interaction effects of four factors on the quality of starting silicon wafers. Chan e Chan (2003) presented a simulation modelling and analysis of a serial production line in a printed circuit board (PCB) factory. Factorial designs also have been applied in simulation techniques to evaluate the performance of existing manufacturing systems to find out active factors that have great impacts on the current operational problems. Nazzal et al. (2006) integrated simulation modeling, factorial design, and economic justification tools to build a comprehensive framework for strategic capacity expansion. Ayanso et al. (2006) used computer simulation and a full factorial experimental design to study and to define Inventory rationing policy.

There are also other important applications of factorial designs in industrial engineering. Bagici and Iş1k (2006) employed factorial designs to investigate the surface roughness when orthogonal cutting tests were carried on unidirectional glass fibre reinforced plastics (GFRP). Lin et al. (2007) used fractional factorial experiments to propose an efficient approach to develop a robust plasma spraying coating process. Datta e Bandyopadhyay (2008) applied factorial designs to evaluate an optimal parameter combination to obtain acceptable quality characteristics of bead geometry in submerged arc bead-on-plate weldment on mild steel plates. Nagesh e Datta (2008) proposed an integrated approach based on the use of Design of Experiments (DOE), Artificial Neural Networks (ANN) and Genetic Algorithm (GA) for modeling Gas Metal Arc Welding (GMAW) processes. Roy et al. (2010) presented a fractional factorial design approach for an inventory model (of a volume flexible manufacturing system for a deteriorating item with randomly distributed shelf life, continuous time-varying demand, and shortages over a finite time horizon) along with its practical implication.

Zhang et al. (2010) investigated important operating variables in the electrochemical treatment of acrylic fiber manufacturing wastewater (AFMW) with boron-doped diamond (BDD) electrode. Jayabal et al. (2010) used factorial design methodology to evaluate mechanical and machinability characteristics of hybrid composites in India. Erginel (2010) applied factorial design to analyse several materials and methods used for packing of products in order to discover the optimum level of packing materials to minimize damage to the product. Galanis e Manolakos (2010) employed factorial design in the development of a surface roughness model for turning of femoral heads from AISI 316L stainless steel. Amari e Mohtashami (2011) presented a multi-objective formulation of the buffer allocation problem in unreliable production lines. Factorial designs also have been used to build a meta-model for estimating production rate based on a detailed, discrete event simulation model. Savic et al. (2012) applied the experimental design principles in pharmaceutical development and discussed the impact of these principles on pharmaceutical legislation. A special kind of factorial designs is given by saturated fractional factorial designs used in industrial applications when we have only a very small number of experiments due to time and costs.

Inferences on the effects of the different factors on the response variable of interest have been explored in the literature using different approaches. Daniel (1959) introduced the graphical method (Q-Q plots) of the half-normal plot to explore the important factors on a response variable of interest; fractional replication was first discussed by Finney (1945). When we have primary interest only on the main effects, we could use saturated factorial designs (e.g., Plackett \& Burman,1946). The use of saturated designs has become very popular for screening factors, especially in industrial applications where the observations usually are very expensive to obtain (see for example, Box et al., 1978; Daniel, 1959; Wu \& Hamada, 2000 ; Cox \& Reid, 2000). This is the case when the number of factors is too large or when we have destructive tests. A saturated design is a fractional factorial design in which the number of parameters in the main effect models is equal to the number of runs. In this paper, we consider saturated designs in which $k=n-1$ main effects are considered in $n$ experimental units or experiments without replicates. In such designs, all information is used to estimate the main effect parameter, leaving no degrees of freedom to estimate the error variance. The classical analysis allows only the estimation of main effects under the assumption that interactions are negligible. A well-known two- 
level saturated design is based on a work developed by Plackett and Burman (1946) where the constructed designs use Hadamard matrices of order $n$, where $n$ is a multiple of 4 .

In this paper, we propose the use of empirical Bayesian methods to analyse data from a saturated design, since the use of saturated classical approach based on least squares estimation usually only permits the estimation of the main effects. According to Miguel (2006), methodologically this study can be classified as pure but with practical applications and objectively descriptive, and taking a quantitative approach. Bertrand and Fransoo (2002) defined quantitative research in production engineering where a problem is modeled whose variables present causal and quantitative relationships. In general, quantitative research uses mathematical, statistical, or computational modeling (simulation) - specifically in this paper, statistical modeling will be adopted.

The paper is organized as follows: in section 2, we introduce a simulated data set from a saturated twolevel design to motivate our approach; in section 3, we introduce a classical analysis for data from a saturated design; in section 4, we introduce a Bayesian analysis assuming conjugated or other priors for the parameters of the model; in section 5, we introduce an empirical Bayesian approach; in section 6, we analyse the data from a saturated design introduced in section 2; finally, in section 7 , we present some concluding remarks

\section{A simulated example}

Consider a 12-run Plackett-Burman design and a simulated data set from the model $\mathrm{Y}=2 \mathrm{x}_{1}+\mathrm{x}_{2}+1.5 \mathrm{x}_{3}+\varepsilon$, where $\varepsilon \sim \mathrm{N}\left(0,0.25^{2}\right)$ and,

$x_{1}=\left\{\begin{array}{cc}-1 & \text { when factor A uses "low" level (-) } \\ 1 & \text { when factor A uses "high" level (+) }\end{array}\right.$

In the same way, we obtain the codes for factors $B$ to $K$. From this model used to simulate the data, we observe that the factors $A, B$ and $C$ are active in the experiment. The simulated data are given in Table 1 (data set introduced by Baba \& Gilmour, 2006). The general model for the data from a two-level saturated design is given by,

$$
Y=\beta_{0}+\beta_{1} x_{1}+\cdots+\beta_{k} x_{k}+\varepsilon
$$

where $x_{i}$ denotes the $\mathrm{i}$-th factor main effect and $\varepsilon \sim \mathrm{N}\left(0, \sigma^{2}\right)$. The model can be expressed in matrix notation by,

$$
\mathrm{E}[\mathbf{y}]=\mathbf{X} \boldsymbol{\beta}
$$

where $\mathbf{X}$ is a $n \times p$ matrix showing the levels at which the factors are fixed, $\beta$ is a $p \times 1$ vector of parameters and $\mathbf{y}$ is $n \times 1$ vector of observations.

\section{Table 1}

\begin{tabular}{|c|c|c|c|c|c|c|c|c|c|c|c|}
\hline \multicolumn{12}{|c|}{ Factor } \\
\hline A & B & $\mathrm{C}$ & $\mathrm{D}$ & $\mathrm{E}$ & $\mathrm{F}$ & $\mathrm{G}$ & $\mathrm{H}$ & I & $\mathrm{J}$ & $\mathrm{K}$ & Response \\
\hline+ & + & - & + & + & + & - & - & - & + & - & 1.7502 \\
\hline+ & - & + & + & + & - & - & - & + & - & + & 2.7130 \\
\hline- & + & + & + & - & - & - & + & - & + & + & 0.4377 \\
\hline+ & + & + & - & - & - & + & - & + & + & - & 4.4825 \\
\hline+ & + & - & - & - & + & - & + & + & - & + & 1.4302 \\
\hline+ & - & - & - & + & - & + & + & - & + & + & -0.5429 \\
\hline- & - & - & + & - & + & + & - & + & + & + & -4.4129 \\
\hline- & + & - & + & + & - & + & + & + & - & - & -2.4942 \\
\hline+ & - & + & + & - & + & + & + & - & - & - & 2.5350 \\
\hline - & + & + & - & + & + & + & - & - & - & + & 0.8998 \\
\hline- & - & - & - & - & - & - & - & - & - & - & -4.6797 \\
\hline
\end{tabular}

A twelve-run Plackett-Burman design 


\section{A classical analysis}

The use of least square estimation permits to obtain estimates for the main effects, but there are no degrees of freedom to estimate the error. This is a great difficult to get inferences from data of a saturated design, that is, the usual analysis of variance (ANOVA) cannot be used. Assuming that the matrix $\mathbf{X}^{\prime} \mathbf{X}$ is nonsingular, as is the case of Plackett-Burman design, the least squares estimates of the main effects are given by,

$$
\tilde{\beta}=\left(X^{\prime} X\right)^{-1} X^{\prime} \mathbf{y} \text {. }
$$

From the simulated data of Table 1 , we get: $\widehat{\beta 0}=0.057 ; \widehat{\beta 1}=2.005 ; \widehat{\beta 2}=1.028 ; \widehat{\beta 3}=1.548 ; \widehat{\beta 4}=0.031$; $\widehat{\beta 5}=0.091 ; \widehat{\beta 6}=0.071 ; \widehat{\beta 7}=0.021 ; \widehat{\beta 8}=-0.069 ; \widehat{\beta 9}=-0.010 ; \widehat{\beta 10}=-0.011$ and $\widehat{\beta 11}=0.031$. The the least squares estimates were obtained using the MINITAB ${ }^{\circledR}$ software. In practical work, usually the researches consider the use of normal plots to decide by the important factors, but the interpretation of the normal plots depends on how strongly the experimenter believes in factor sparsity. A possible alternative to analyse data from saturated two-level designs is the use of Bayesian methods.

\section{A Bayesian analysis}

The likelihood function for $\boldsymbol{\beta}$ and $\sigma^{2}$ is given by,

$\mathrm{f}\left(\mathrm{y} \mid \boldsymbol{\beta}, \sigma^{2}\right)=\left(2 \pi \sigma^{2}\right)^{-\mathrm{n} / 2} \exp \left\{-(\mathrm{y}-\mathrm{X} \boldsymbol{\beta})^{\prime}(\mathrm{y}-\mathrm{X} \boldsymbol{\beta}) / 2 \sigma^{2}\right)$

Expanding the quadratic form $(y-X \beta)^{\prime}(y-X \beta)$ in (4), we have,

$\mathrm{f}\left(\mathrm{y} \mid \boldsymbol{\beta}, \sigma^{2}\right)=\left(2 \pi \sigma^{2}\right)^{-\mathrm{n} / 2} \exp \left\{-(\boldsymbol{\beta}-\hat{\boldsymbol{\beta}})^{\prime} \mathbf{X}^{\prime} \mathbf{X}(\boldsymbol{\beta}-\hat{\boldsymbol{\beta}}) / 2 \sigma^{2}+\mathrm{Q}\right)$

where $\hat{\boldsymbol{\beta}}$ is given by Eq. (3) and $\mathrm{Q}=(\mathrm{y}-\mathrm{X} \boldsymbol{\beta})^{\prime}(\mathrm{y}-\mathrm{X} \boldsymbol{\beta})$ is the residual sum of squares.

Note that for saturated designs, we can estimate the vector of parameter $\boldsymbol{\beta}$, but the quantity $\mathbf{Q}$ is always zero, which means that the error variance cannot be estimated from the likelihood and the analysis used in the general linear model is not appropriate for saturated designs. In this way, we assume a Bayesian approach to analyse data from a saturated design.

Different priors could be considered to analyse data from saturated designs (see Baba and Gilmour, 2006), but since data from saturated designs provide only limited information, the interpretation of these data depends heavily on the prior assumptions.

Among the different priors considered by these authors, a conjugate prior is given (from (5) ) by,

$\mathrm{f}\left(\boldsymbol{\beta}, \sigma^{2}\right)=(\mathrm{a} / 2)^{\mathrm{d} / 2}\left(\sigma^{2}\right)^{-(\mathrm{d}+\mathrm{p}+2) / 2} /\left((2 \pi)^{-1 / 2}|\mathrm{~V}|^{1 / 2} \Gamma(\mathrm{d} / 2) \cdot \exp \left\{-(\boldsymbol{\beta}-\mathbf{m})^{\prime} \mathrm{V}^{-1}(\boldsymbol{\beta}-\mathbf{m}) / 2 \sigma^{2}+\mathrm{a}\right)\right.$

with hyperparameters $\mathrm{a}>0, \mathrm{~d}>0, \mathrm{~m} \in \mathrm{R}^{\mathrm{p}}$ and $\mathrm{V}$ is a $\mathrm{p} \times \mathrm{p}$ positive definite matrix (a Normal - Inverse Gamma distribution). Assuming different values for the hiperparameters of the prior (6), the use of conjugate priors are very inflexible since very informative priors can lead to the posterior being very vague and centered in the wrong place (see Baba \& Gilmour, 2006).

Other priors as a finite mixture of densities or non-conjugate priors are also considered in the literature, but the obtained posterior summaries are heavily dependent of the choice of the prior for $\boldsymbol{\beta}$ and $\sigma^{2}$.

Observe that in a saturated design, we are using $n$ observations to estimate $n+1$ parameters, that is, in absence of any prior knowledge, the data do not provide any information. 


\section{Use of empirical Bayesian methods}

To get some information for the variance $\sigma^{2}$ of the error for data of a saturated design, let us assume the following procedure: among the $g$ experiments considered in the saturated two-level design, we have for each factor, $g / 2$ values in the "high" level (+) and $g / 2$ values in the "low" level (-). From these $g / 2$ values in each level "+" or "-", we get its standard deviations denoted by $\mathbf{s}_{\ell_{+}}$and $\mathbf{s}_{\ell_{-}}, 1=1,2, \ldots, \mathrm{K}$. Denoting by $\mathbf{y}_{\ell i}^{+}$and the $\mathrm{g} / 2$ values in the "high" level + for factor 1, we have,

$$
\left(s_{l+}\right)^{2}=\left\lfloor\frac{1}{0.5 g-1}\right\rfloor \sum_{I=1}^{g / 2}\left[y_{l i}^{+}-U^{*}\right]^{2}
$$

where,

$U^{*}=\left[\frac{1}{0.5 g}\right] \sum_{I=1}^{g / 2} y_{l i}^{+}, \quad l=1, \cdots K$.

In the same way, denoting by $\mathrm{y}_{\boldsymbol{\ell}_{i}}^{-}$the $\mathrm{g} / 2$ values in the "low" level (-) of factor 1 ,

$$
\left(s_{l-}\right)^{2}=\left\lfloor\frac{1}{0.5 g} \sum_{I=1}^{g^{\prime \prime}}\left(y_{l i}^{-}-U^{* *}\right)^{2}\right.
$$

where,

$$
U^{* *}=\left[\frac{1}{0.5 g}\right] \sum_{I=1}^{g / 2} y_{l i}^{-}, l=1, \cdots, K .
$$

In this way, we have $K$ values $\mathrm{s}_{\ell+}^{2}$ and $\mathrm{s}_{\ell_{-}}^{2}$, that is, a total of $2 K$ sample variances, or $2 K$ quantities.

From these $2 \mathrm{~K}$ quantities, we get the sample mean denoted by $\mathrm{A}$ and the sample variance denoted byB $^{2}$, which can be used to find appropriated values for the hiperparameters of the prior distribution for the variance $\sigma^{2}$ of the error in Eq. (1). Assuming a gamma prior distribution for $\sigma^{2}$, that is,

$\sigma^{2} \sim \operatorname{Gamma}(\mathrm{a}, \mathrm{b})$

where $E\left[\sigma^{2}\right]=a / b$ and $\operatorname{var}\left[\sigma^{2}\right]=a / b^{2}$, we get values for the hyperparameters $a$ and $b$ by solving the following equations:

$a / b=A$,

$a / b^{2}=B^{2}$.

\section{Analysis of the data of Table 1 - Discussion}

Let us assume the simulated data of Table 1 considering a 12-run Plackett-Burman design with model $\mathrm{Y}=2 \mathrm{x}_{1}+\mathrm{x}_{2}+1.5 \mathrm{x}_{3}+\varepsilon$, where $\varepsilon \sim \mathrm{N}\left(0,0.25^{2}\right)$.

Considering the $g=12$ observations in factor $A$, we have 6 observations in level "high" or "+" given by $1.7502,2.7130,4.4825,1.4302,-0.5429$ and 2.5350 , from where we get $\mathrm{s}_{1+}=1.661$ (see (7)). In the same way, considering the 6 observations in factor A in the level "low" or "-" we have 0.4377, $4.4129,-1.4384,-2,4942,0.8998$ and -4.6797 , and $\mathrm{s}_{1-}=2.362$ (see (8)). With the same approach, we get for other factors B to $\mathrm{K}$ the values $\mathbf{s}_{\ell_{+}}$and $\mathbf{s}_{\ell_{-}}, \quad 1=2,3, \ldots, 11$. In Table 2, we have these 22 quantities $\left(\mathbf{S}_{\ell_{+}}, \mathbf{S}_{\ell_{-}}\right)$for the 11 factors. 
Table 2

Estimated quantities $\mathbf{S}_{\ell_{+}}$and $\mathbf{S}_{\ell_{-}}$

\begin{tabular}{ccccccccccc}
\hline $\mathrm{A}$ & $\mathrm{B}$ & $\mathrm{C}$ & $\mathrm{D}$ & $\mathrm{E}$ & $\mathrm{F}$ & $\mathrm{G}$ & $\mathrm{H}$ & $\mathrm{I}$ & $\mathrm{J}$ & $\mathrm{K}$ \\
\hline 1.661 & 2.252 & 2.073 & 2.920 & 1.984 & 2.600 & 3.260 & 1.857 & 3.380 & 3.010 & 2.450 \\
2.362 & 3.220 & 2.820 & 3.070 & 3.740 & 3.350 & 2.710 & 3.810 & 2.560 & 2.990 & 3.460 \\
\hline
\end{tabular}

Considering the squares for the 22 quantities given in Table 2, we get a sample mean $A=8.311$ and a sample variance $\mathrm{B}^{2}=3.260$. From Eq. (10), we get the values of the hyperparameters of the gamma prior distribution given in Eq. (9) for the variance $\sigma^{2}$ of the error in Eq. (1), given by $a=6.4994$ and $b=0.7820$. Also assuming normal $\mathrm{N}\left(0,10^{6}\right)$ priors for the regression parameters $\beta_{\ell}=0,1, \ldots, 11$ in Eq. (1) (that is, very non-informative priors), we use Markov Chain Monte Carlo (MCMC) methods to get the posterior summaries of interest (see for example, Chib \& Greenberg, 1995 or Gelfand \& Smith, 1990).

Using the Winbugs software (Spiegelhalter et al., 2004) we simulated 5,000 Gibbs samples (taking every $10^{\text {th }}$ sample) of the joint posterior distribution for $\beta$ and $\sigma^{2}$, after a "burn-in period" of size 5,000 . Convergence of the Gibbs sampling algorithm was monitored by observing the traceplots of the simulated samples for each parameter. In Table 3, we have the posterior summaries of interest. In Table 4 , we have the observed and predicted values $\hat{y}=\hat{\beta}_{0}+\hat{\beta}_{1} x_{1}+\cdots+\hat{\beta}_{11} x_{11}$, where $\hat{\beta}_{l}$ are the Monte Carlo estimates of the posterior means for $\beta_{l}, l=0, \cdots, 11$ based on the 5000 simulated Gibbs samples given in Table 3, assuming a Gamma $(6.4994 ; 0.7820)$ prior for $\sigma^{2}$ and normal $\mathrm{N}\left(0,10^{6}\right)$ priors for $\beta_{l}, l=0$, $2, \ldots, 11$ (use of the empirical Bayesian method). Let us denote this model as "model 1". From the results of Table 4, we observe very good predictions assuming "model 1", that is, good inferences for the data from a saturated two-level design. In Table 4, we also have the predicted values considering other priors for $\sigma^{2}$ and normal $\mathrm{N}\left(0,10^{6}\right)$ priors for $\beta_{1}$ also considering the use of the Winbugs software (5,000 Gibbs samples after a "burn-in period" of size 5,000). Another possibility is to assume a uniform $\mathrm{U}(0,1000)$ prior for $\sigma^{2}$ and normal $\mathrm{N}\left(0,10^{6}\right)$ priors for $\beta_{\ell}$. Let us denote this model as "model 2". A third model denoted as "model 3 ", is considered assuming a uniform $\mathrm{U}(0,1000)$ prior for $\sigma^{2}$ and $\mathrm{N}\left(\tilde{\beta}_{\ell}\right.$, 0.01 ) priors for $\beta_{\ell}$, where $\tilde{\beta}_{\ell}$ are the least squares estimates for $\beta_{1}, 1=0,2, \ldots, 11$ (see section 3 ), that is, very informative priors for the regression parameters, but very non-informative prior for $\sigma^{2}$. From the results of Table 4, we observe that the predicted values assuming "model 2" and "model 3" are very different of the observed values, that is, we get bad predictions.

Table 3

Posterior summaries (use for empirical Bayesian methods)

\begin{tabular}{cccc}
\hline parameter & posterior mean & S.D & $95 \%$ cred.interval \\
\hline$\beta_{0}$ & 0.0694 & 0.8030 & $(-1.5270 ; 1.6810)$ \\
$\beta_{1}$ & 2.0180 & 0.8290 & $(0.3696 ; 3.7140)$ \\
$\beta_{2}$ & 1.0270 & 0.8262 & $(-0.6176 ; 2.6250)$ \\
$\beta_{3}$ & 1.5490 & 0.8387 & $(-0.1182 ; 3.2030)$ \\
$\beta_{4}$ & 0.0291 & 0.8174 & $(-1.5900 ; 1.6920)$ \\
$\beta_{5}$ & 0.1135 & 0.8308 & $(-1.5220 ; 1.7120)$ \\
$\beta_{6}$ & 0.0488 & 0.8408 & $(-1.6250 ; 1.6880)$ \\
$\beta_{7}$ & 0.0153 & 0.8458 & $(-1.6420 ; 1.7150)$ \\
$\beta_{8}$ & -0.0729 & 0.8198 & $(-1.6390 ; 1.5540)$ \\
$\beta_{9}$ & -0.0248 & 0.8231 & $(-1.7390 ; 1.587090)$ \\
$\beta_{10}$ & -0.0320 & 0.8441 & $(-1.6180 ; 16650)$ \\
$\beta_{11}$ & 0.02595 & 0.8245 & $(0.0634 ; 0.3151)$ \\
\hline
\end{tabular}


Table 4

Observed and predicted values

\begin{tabular}{cccc}
\hline Observed & Predicted "model 1" & Predicted "model 2" & Predicted "model 3" \\
\hline 1.7502 & 1.7810 & -4.3830 & -4.6797 \\
2.7180 & 2.7940 & 6.7420 & -4.4129 \\
0.4377 & 0.4249 & -0.5821 & -2.4942 \\
4.4825 & 4.4770 & 11.7900 & -1.4384 \\
1.4302 & 1.4160 & 11.7500 & -0.5429 \\
-0.5429 & -0.4920 & -15.5700 & 0.4377 \\
4.4129 & -4.5030 & -11.0800 & 0.8998 \\
-1.4384 & -1.4640 & 12.0600 & 1.4302 \\
-2.4942 & -2.4530 & 12.1200 & 1.7502 \\
2.5350 & 2.5470 & 8.6140 & 2.5350 \\
0.8998 & 0.9318 & -13.9600 & 2.7130 \\
-4.6792 & -4.6270 & 10.1800 & 4.4825 \\
\hline
\end{tabular}

From these results, we observe that the use of the proposed empirical Bayesian method could be a powerful methodology for applications of saturated two-level designs. It is also important to point out that the obtained $95 \%$ credible intervals considering "model 1" (empirical Bayesian model) show that the factors $x_{1}, x_{2}$ and $x_{3}$ are active in the experiment (in agreement to the true values of $\beta_{1}, \beta_{2}$ and $\beta_{3}$ given by the model $\mathbf{E}[\mathbf{Y}]=2 \mathbf{x}_{1}+\mathbf{x}_{2}+1.5 \mathbf{x}_{3}$ used to simulate the data) and very accurate posterior means for $\beta_{1}, \beta_{2}$ and $\beta_{3}$.

\section{Concluding Remarks}

The use of saturated factorial designs has been extensively used by industrial researchers and engineers as a powerful methodology for screening factors, especially in the presence of a great number of factors. Usually, we get some information about important factors on the response variable of interest using normal Q-Q plots. The use of these normal plots in the interpretation of factorial two-level experiments usually could be very subjective and in many cases where we are in trouble to find the active factors on the response of interest. The use of the empirical Bayesian approach introduced in this paper could be of great interest in applications. We also observed very good predictions and inferences for the parameters of interest using our proposed methodology.

\section{References}

Amari, M., \& Mohtashami, A. (2012). Buffer allocation in unreliable production lines based on design of experiments, simulation, and genetic algorithm. The International Journal of Advanced Manufacturing Technology, 62, 371-383.

Ayanso, A., Diaby, M., \& Nair, S.K. (2006). Inventory rationing via drop-shipping in Internet retailing: A sensitivity analysis. European Journal of Operational Research, 171, 135-152

Baba, M. Y., \& Gilmour, S. G. (2006). Bayesian estimation from saturated factorial designs, in: Colosimo, B. M.; Castilho E., Bayesian process monitoring, control and optimization. Chapman \& Hall, 311-322.

Bagci, E., \& Işık, B. (2006). Investigation of surface roughness in turning unidirectional GFRP composites by using RS methodology and ANN. The International Journal of Advanced Manufacturing Technology, $31,10-17$.

Bertrand, J.W., \& Fransoo, J.C. (2002). Operations management research methodologies using quantitative modelling. International Journal of Operations \& Production Management, 22, 241-26.

Box, G. E. P., Hunter, W. G., \& Hunter, J. S. (1978). Statistics for Experimenters: an Introduction to Design, Analysis and Model Building, New York: John Wiley \& Sons.

Chan, F. T. S., \& Chan, H. K. (2003). Simulation analysis of a PCB factory using factorial design - A case study. International Journal of Advanced Manufacturing Technology, 21, 523-533

Chib, S., \& Greenberg, E. (1995). Understanding the Metropolis-Hastings algorithm. The American Statistician, 49(4), 327-335.

Cox, D.R., \& Reid, N. (2000). The Theory of the Design of Experiments. London: Chapman and Hall. 
Daniel, C. (1959). Use of half-normal plot in interpreting factorial two-level experiments. Technometrics, 1(4), 11-49.

Datta, S., Bandyopadhyay, A., \& Pal, P. K. (2008). Modeling and optimization of features of bead geometry including percentage dilution in submerged arc welding using mixture of fresh flux and fused slag. The International Journal of Advanced Manufacturing Technology ,36, 1080-1090

Erginel, N. (2010). Modeling and analysis of packing properties through a fuzzy inference system. Journal of Intelligent Manufacturing, 21, 869-874.

Feng, C., Wang, X., \& Yu, Z. (2003). Neural networks modeling of honing surface roughness parameters defined by IS0 13565. Journal of Manufacturing Systems, 21(5), 395-408.

Finney, D.J. (1945). The fractional replication of factorial arrangements. Annals of Eugenics, 12, 283-290.

Fisher, R.A. (1926). The arrangement of field experiments. Journal of Ministry of Agriculture, 33, 503-513.

Fisher, R.A. (1935). Design of experiments. Edinburgh: Oliver \& Boyd.

Galanis, N.I., \& Manolakos, D. E. (2010). Surface roughness prediction in turning of femoral head. The International Journal of Advanced Manufacturing Technology, 51, 79-86.

Gelfand, A. E., \& Smith, A. F. M. (1990). Sampling-based approaches to calculating marginal densities. Journal of the American Statistical Association, 85, 410, 398-409.

Jayabal, S., Natarajan, U., \& Sekar, U. (2011). Regression modeling and optimization of machinability behavior of glass-coir-polyester hybrid composite using factorial design methodology. The International Journal of Advanced Manufacturing Technology, 55, 263-273.

Kleijnen, J. P. C., \& Standridge, C. R. (1988). Experimental design and regression analysis in simulation: An FMS case study. European Journal of Operational Research, 33, 257-261.

Lin, B, Jean, M, \& Chou, J. (2007). Using response surface methodology with response transformation in optimizing plasma spraying coatings. The International Journal of Advanced Manufacturing Technology, 34, 307-315.

Miguel, P.A.C. (2007). Estudo de caso na engenharia de produção: estruturação e recomendações para sua condução. Produção, 17, 216-229.

Nagesh, D. S., \& Datta, G. L. (2008). Modeling of fillet welded joint of GMAW process: integrated approach using DOE, ANN and GA. The International Journal on Interactive Design and Manufacturing, 2, 127-136.

Nazzal, D., Mollaghasemi, M., \& Anderson, D. (2006). A simulation-based evaluation of the cost of cycle time reduction in Agere Systems wafer fabrication facility - a case study. The International Journal of Production Economics, 100, 300-313.

Pei, Z.J., Xin, X.J., \& Liu, W. (2003). Finite element analysis for grinding of wire-sawn silicon wafers: a designed experiment. International Journal of Machine Tools \& Manufacture, 43, 7-16.

Philpott, M.L., Warrington, C.S., Branstad, E.A., David, R., \& Nita, R.P. (1996). A Parametric ContractModeler for DFM Analysis. Journal of Manufacturing Systems, 15(4), 256-267.

Plackett, R. L., \& Burman, J. P. (1946). The design of optimum multifactorial experiments. Biometrika, 33, 305-325.

Roy, A., Kar, S., \& Manoranjan, M. (2010). A volume flexible production-policy for randomly deteriorating item with trended demand and shortages. The International Journal of Production Economics, 128, 188-199

Savic, I. M., Marinkovic, V.D., Tasic, L., Krajnovic, D., \& Savic, I. M. (2012). From experimental design to quality by design in pharmaceutical legislation. Accreditation and Quality Assurance, 17, 627-633.

Spiegelhalter, D., Thomas, A., Best, N., \& Lunn, D. (2004) Winbugs user manual, version 1.4. Available: http://www.mrc-bsu.cam.ac.uk/bugs. [Acessed 8 december 2012].

Wu, C.F.J., \& Hamada, M. (2000). Experiments: planning, analysis and parameter design optimization. New York: John Wiley \& Sons.

Yates, F. (1935). Complex experiments (with discussion). Supplement. Journal of the Royal Statistics Society, 2, 181-247.

Yates, F. (1937). The design and analysis of factorial experiments. Technical communication 35. Harpenden: Imperial Bureau of Soil Science.

Zhang, C., Wang, J., Zhou, H., Fu, D., \& Gu, Z. (2010). Anodic treatment of acrylic fiber manufacturing wastewater with boron-doped diamond electrode: A statistical approach. Chemical Engineering Journal, 161, 93-98. 